hyphae of S. commune, which can be seen under the electron microscope as a layer of interwoven rodlets ${ }^{4,5}$. Targeted disruption of the $S C 3$ gene produces mutants $(\triangle S C 3)$ that are unable to form aerial hyphae ${ }^{6}$.

SC3 is secreted as a monomer, but when it encounters an air-water interface or an interface with a hydrophobic surface, it aggregates into a large polymeric complex ${ }^{4}$. This hydrophobin polymer forms a thin layer that is hydrophobic on the side where it is decorated with rodlets, and hydrophilic on the other. This alteration in structure of SC3 occurs in response to changing environmental conditions encountered by the fungus as it grows from water into the air, allowing the fungus to produce hydrophobic aerial hyphae.

But this is only half of the story. SC3 is produced abundantly by $S$. commune even when the fungus is growing in liquids ${ }^{2,4}$ and the importance of this is now clear. In a sim-

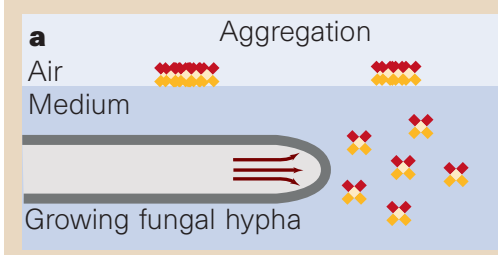

b
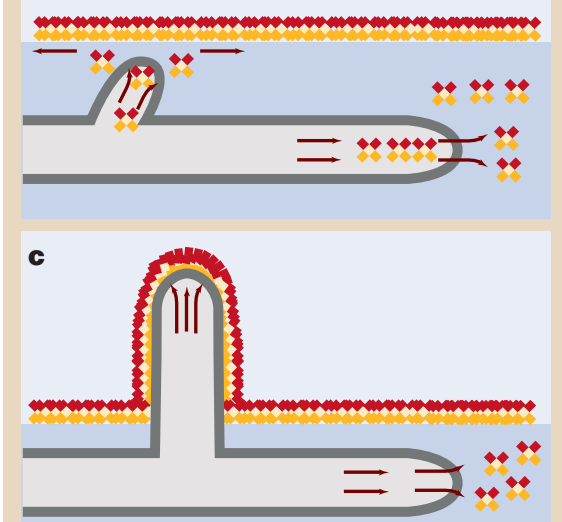

Hydrophobic domains Hydrophilic domains Hydrophobin monomer

Figure 2 Model for the emergence of aerial hyphae based on the experiments of Wösten et al. ${ }^{1}$ with the gill mushroom $S$. commune. a, A hypha growing in its normal damp environment, surrounded by a film of water. During growth, SC3 hydrophobin is secreted in abundance. SC3 monomers are depicted as having two hydrophobic domains and two hydrophilic domains ${ }^{2}$ and aggregate at the air-water interface into an amphipathic (with a hydrophobic and a hydrophilic face) layer. b, This causes a dramatic reduction in surface tension, allowing the hypha to escape and grow into the air. Hydrophobin secretion continues.

c, The aerial hypha continues to secrete SC3 hydrophobin, which forms a hydrophobic rodlet layer on the outside of the cell wall. ple experiment, Wösten et al. showed that the normal surface tension of water, $72 \mathrm{~mJ}$ $\mathrm{m}^{-2}$, can be reduced to as low as $24 \mathrm{~mJ} \mathrm{~m}^{-2}$ by adding purified SC3. This makes SC3 the most powerful surface-active protein known and suggests that secretion of hydrophobins at high concentrations dramatically lowers the surface tension, allowing hyphae to escape the liquid and grow into the air. Consistent with this, the surface tension of a liquid culture of S. commune falls by a similar amount during growth of the fungus; and when purified SC3 is added to culture medium surrounding a $\Delta S C 3$ mutant, the mutant becomes able to put out aerial hyphae.

Aerial hyphae in $\Delta S C 3$ mutants that have been treated with exogenous SC3 protein are hydrophilic, indicating that continued secretion of SC3 from aerial hyphae is required for the formation of a hydrophobic surface layer. Thus SC3 has two functions. First, it lowers the surface tension of the water that surrounds the hyphae, allowing them to emerge from the liquid; and second, it coats aerial hyphae with a hydrophobic wall that enables them to grow into the air (Fig. 2) and probably to withstand desiccation.

Since they were first identified in the early 1990s, hydrophobins have been found in many filamentous fungi and may well be ubiquitous $^{2}$. Hydrophobin genes are absent, however, from the genome of the unicellular fungus Saccharomyces cerevisiae. The evolution of hydrophobins may therefore have been concurrent with the divergence of the filamentous fungi and, in particular, their colonization of terrestrial ecosystems.

There is still much to be learned about these unusual proteins. Certain fungal species, for example, produce a variety of hydrophobins with diverse biochemical properties ${ }^{2}$, indicating that, in addition to their role in aerial growth, hydrophobins may participate in many other developmental processes. The structural biology of hydrophobins also remains largely unexplored. How, for example, do these small proteins aggregate in response to air-water interfaces, and what conformation do hydrophobin monomers adopt within a rodlet layer? Wösten et al. have reached an important milestone in showing how hydrophobins bring about aerial growth in fungi: now we need to determine the molecular basis of their activity.

Nicholas J. Talbot is in the School of Biological

Sciences, University of Exeter, Washington Singer Laboratories, Perry Road, Exeter EX4 4QG, UK. e-mail: N.J.Talbot@exeter.ac.uk

1. Wösten, H. A. B. et al. Curr. Biol. 9, 85-88 (1999).

. Wessels, J. G. H. Adv. Microbial Physiol. 38, 1-45 (1997)

Kershaw, M. J., Wakley, G. E. \& Talbot, N. J. EMBO J. 17, 3838-3849 (1998)

4. Wösten, H. A. B., Schuren, F. H. J. \& Wessels, J. G. H. Plant Cell 5, 1567-1574 (1993).

5. Wösten, H. A. B., Ásgeirsdóttir, S. A., Krook, J. H., Drenth, J. H. H. \& Wessels, J. G. H. Eur. J. Cell Biol. 63, 122-129 (1994) 6. van Wetter, M. A., Schuren, F. H. J. \& Wessels, J. G. H. FEMS Microbiol. Lett. 140, 265-270 (1996).
Daedalus

\section{Press-fit chemistry}

Many catalysts provide a passive surface or cavity on which reacting molecules can sit in the right position to react together. Daedalus is now dreaming up a more active type of catalyst.

He recalls the catalytic zeolites, which are riddled with molecular-scale cavities, within which entering molecules can react. Sometimes the cavities actually expand when molecules enter them. So, says Daedalus, imagine an elastic porous catalyst, perhaps a piezoelectric zeolite or microporous silica, expanding and contracting under external control. In its expanded state, it allows reacting molecules to enter its cavities freely. Then it contracts, squashing them together in a way that forces them to react. On the next expansion, the product molecule can escape, to be replaced by new reagents. The cycle could be repeated at megahertz rates.

DREADCO chemists are now trying it. They are combining the skills of the piezoelectric quartz crystal and silicabased catalyst industries to study the effects of setting such catalysts into intense vibration. With luck, the chemical yields of standard test reactions should increase dramatically. Even better, a piezoelectric catalyst could be 'tuned' electronically by setting it into different vibrational modes. A mode which elongated its molecular sites or cavities would give linear molecules; one which flattened them would encourage planar ones. A single catalyst could generate a wide range of products under perfect control.

But the real goal of the project is to find entirely new reactions, yielding hitherto unknown products. Imagine, says Daedalus, a piezoelectric porous catalyst whose cavities can expand to accept three dinitrogen molecules arranged as a hexagon. When such a cavity contracts, the molecules will be squashed together to form the hitherto unknown hexagonal molecule $\mathrm{N}_{6}$. Being isoelectronic with benzene, it could well be stable. On the next expansion, it would be released, and more dinitrogen taken up. $\mathrm{O}_{6}$, tetrahedrane (dimerized from acetylene) and many other unknown molecules, could be made in the same way.

$\mathrm{N}_{6}$ would probably be a powerful high explosive, $\mathrm{O}_{6}$ a ferocious oxidizing agent, and tetrahedrane an energetic rocket fuel. But they are just the start of a new era of chemical synthesis. A whole bonanza of weird, warped, incredible molecules could pour from the new cornucopia of piezoelectric catalysis.

David Jones 